Andrews University

Digital Commons @ Andrews University

Faculty Publications

$6-1-2000$

\title{
A Review of Empirical Studies Assessing Ethical Decision Making in Business
}

Terry W. Loe

Hankamer School of Business

Linda K. Ferrell

The University of Tampa

Phylis Mansfield

Andrews University, mansfiel@andrews.edu

Follow this and additional works at: https://digitalcommons.andrews.edu/pubs

Part of the Business Law, Public Responsibility, and Ethics Commons

\section{Recommended Citation}

Loe, Terry W.; Ferrell, Linda K.; and Mansfield, Phylis, "A Review of Empirical Studies Assessing Ethical Decision Making in Business" (2000). Faculty Publications. 2541.

https://digitalcommons.andrews.edu/pubs/2541

This Article is brought to you for free and open access by Digital Commons @ Andrews University. It has been accepted for inclusion in Faculty Publications by an authorized administrator of Digital Commons @ Andrews University. For more information, please contact repository@andrews.edu. 


\section{A Review of Empirical Studies Assessing Ethical Decision Making in Business}

\begin{abstract}
This article summarizes the multitude of empirical studies that test ethical decision making in business and suggests additional research necessary to further theory in this area. The studies are categorized and related to current theoretical ethical decision making models. The studies are related to awareness, individual and organizational factors, intent, and the role of moral intensity in ethical decision making. Summary tables provide a quick reference for the sample, findings, and publication outlet. This review provides insights for understanding organizational ethical decision constructs, where ethical decision making theory currently stands, and provides insights for future empirical work on organizational ethical decision making.
\end{abstract}

Terry W. Loe is Assistant Professor of Marketing, at Baylor University and Associate Director of the Baylor University Center for Professional Selling. His research interests include ethics in marketing, professionalism in sales and sales management, and developing more efficient work climates in organizations. His research has appeared in the Journal of Contemporary Business Issues, and Proceedings of the American Marketing Association, the Southern Marketing Association and other regional conferences.

Linda K. Ferrell is a Research Affiliate of the University of Tampa Center for Ethics. She has published articles in The Journal of Business Ethics, Journal of Public Policy and Marketing, Case Research Journal and Journal of Marketing Management. Her current research focuses on evaluating the effectiveness of ethics training approaches, international business ethics issues and practices, and the impact of regulation on the area of business ethics.

Phylis Mansfield is Assistant Professor of Marketing at Andrews University and has a background in the financial services industry, and worked as a product manager and business analyst in the banking industry. Her research interests include Consumer Behavior issues, consumer ethics, and business ethics.

\section{Introduction}

Current ethical decision making models present numerous variables that influence ethical choice, providing a theory base for how ethical decisions are made in organizations. There is general agreement among scholars concerning the individual variables and organizational learning processes that influence ethical decisions. The positive ethical decision making models (Ferrell and Gresham, 1985; Hunt and Vitell, 1986; Jones, 1991; Trevino, 1986) and their theoretical underpinnings identify key constructs that assist in understanding the factors that have the greatest effect on an individual's ethical decision making in organizations. Criticisms of normative models of business ethics, which often assume absolute truths about appropriate decision making, led to the development of positive perspectives and models. Positive models describe what actually occurs in the organization, versus normative models that address what should occur. Unlike normative models that specify decision rules for how to make an optimum or correct decision, positive models are more readily evaluated, using scientific modes of inquiry (Thorne and Ferrell, 1993).

Insights into the strength of positive models of ethical decision making come from empirical study that has assessed these relationships. Positive models guide or increase our understanding of business phenomena (Hunt, 1991). Hunt continues, "Scientific knowledge thus rests on the bedrock of empirical testability" (1991, p. 197). In 1994, Ford and Richardson published a comprehensive review of the empirical literature related to ethical beliefs and decision making. This project updates their work by evaluating 
empirical studies in organizational settings and relating these studies to the Jones (1991) Synthesis of Ethical Decision MakingDecision Making Model. Summaries of the findings of these empirical studies are provided along with suggestions for future research.

\section{Theoretical models of organizational ethical decision making}

The Jones model (1991) provides the most comprehensive synthesis model of ethical decision making. The model integrates previous ethical decision making models and represents overall agreement regarding the variables that influence ethical decision making and introduces the concept of "moral intensity." Jones believed previous studies failed to consider the nature of the ethical issue. Moral intensity is the "extent of issue-related moral imperative in a situation" (Jones, 1991, p. 372). The foundation of Jones' model lies in Rest's (1986) four-stage process: recognizing moral issues, making moral judgments, establishing moral intent, and engaging in moral behavior. Jones (1991) uses Rest's (1986) four stages to link the positive ethical decision making models and assumes that ethical choices are not just individual decisions, but are determined by social learning in the organization.

"Recognizing an issue" is encompassed in the works of Ferrell and Gresham (1985), Hunt and Vitell (1986), and Trevino (1986). Trevino (1986) and Rest (1986), who discuss moral evaluation via moral philosophy (deontological and teleological), support "making a moral judgment." Ferrell and Gresham (1985) established that "moral intent" of the individual is moderated by significant others, individual moderators, and opportunity. Trevino (1986) identified both individual and situational moderators as affecting the relationship between making a moral judgment and engaging in moral behavior. The most comprehensively examined variables are discussed below and include gender, moral philosophy, education and work experience (individual factors), culture and climate, codes of ethics (organizational factors), awareness, rewards and sanctions, and significant others (organizational factors). Other studies with less empirical examination are discussed in a miscellaneous category section, and include individual factors: cognitive moral development, moral philosophy, gender, age, education and work experience, nationality, religion, locus of control, and intent; the organizational factor opportunity, and moral intensity.

\section{Empirical studies of ethical decision making in business}

Studies addressing the ethical decision making process in business can be categorized into two distinct pursuits: 1) studies that directly examine the hypotheses set forth by ethical decision making models, and 2) studies identifying the moderators of ethical decision making within the organization. Table I provides a summary of empirical studies of ethical decision making in business by general category of inquiry. Table II provides a summary of the empirical studies that address the direct linkages in ethical decision making models (awareness, individual factors and intent). Table III details the studies of the moderating factors of ethical decision making in the organization (organizational factors such as organizational culture, opportunity, codes, significant others, etc.) and moral intensity. The overall findings of these studies are reviewed with the most comprehensively researched areas discussed first.

\section{A. Gender in ethical decision making}

Table I reviews the studies related to each established dimension of ethical decision making and reveals that the bulk of empirical studies address individual factors that influence ethical decision making. These types of inquiries accompany most ethics studies as control mechanisms and therefore, generate a greater volume of findings than any other single area. Particularly, the role of gender in ethical decision making has received significant examination (twenty-six studies in 
TABLE I

Number of empirical studies of ethical decisionmaking in business by category

\begin{tabular}{lc}
\hline Construct & $\begin{array}{c}\text { \# of empirical } \\
\text { studies }\end{array}$ \\
\hline Awareness & 15 \\
Individual factors & \\
Cognitive moral development & 6 \\
Moral philosophy & 21 \\
Gender & 26 \\
Age & 15 \\
Education and work experience & 18 \\
Nationality & 10 \\
Religion & 3 \\
Locus of control & 4 \\
Intent & 4 \\
Organizational factors & \\
Opportunity & 3 \\
$\quad$ Codes of Ethics & 17 \\
Rewards and sanctions & 15 \\
Culture and climate & 18 \\
Significant others & 11 \\
Moral intensity & 2 \\
\hline
\end{tabular}

business). The bulk of studies either determined no significant gender differences or found females tend to be more ethically sensitive than males. Even though gender is one of the most researched or tangentially researched areas, the findings are mixed and inconclusive. More reflection on the methodology of these studies is necessary; how was ethical behavior measured, what was the composition of the study, and where was the study conducted? The bulk of gender related studies were reported in the Journal of Business Ethics (seventeen) and of the twenty-six studies conducted, eleven used a student sample. Perhaps the use of student samples to measure organizational ethics related issues should be examined. Student samples often are younger than organizational samples and have some imbedded characteristics associated with the sample's inexperience both in life and in the workplace.

\section{B. Moral philosophy}

Moral philosophy ranks second in number of studies conducted (twenty-one). Evaluations of moral philosophy range from examining deontological perspectives versus teleological (Hunt and Vasquez-Parraga, 1993; Mayo and Marks, 1990) to Machiavellianism's influence on ethical decision making (Cyriac and Dharmaraj, 1994; Hegarty and Sims, 1978). Student or faculty samples were utilized in one third of the studies and thirteen appeared in the Journal of Business Ethics. Generally, these studies reveal that moral philosophy is related to ethical decision making and individuals may decide upon using different philosophies based upon experience (early career versus later) or based upon industry. Moral philosophy has not been systematically related to the level of ethical behavior in ethical decision making, which represents opportunity for future research.

\section{Education, work experience and culture and climate}

Eighteen studies explored education and work experience and culture and climate. Education and work experience were found to have negligible or no influence on ethical decision making in half of the studies. The other nine studies produced mixed findings, while some of the studies indicated that higher educational levels are associated with greater ethical sensitivity. The mixed nature of the findings suggests we do not clearly understand the role of experience and education in ethical decision making in organizations. Similar studies of age related to ethical decision making found a positive correlation between age and ethical decision making. Additional research can assist in understanding the role of education, intra-company experience, and cross industry and multi-company experience on ethical decision making. Culture and climate have been found to be pervasive in influencing and adapting organizational ethics. Findings in this area strongly support the theoretical and managerial beliefs that managing 
TABLE II

Empirical research of ethical decision-making in business

\begin{tabular}{|c|c|c|}
\hline Yr./Author: Journal & N: Sample composition & Findings \\
\hline \multicolumn{3}{|c|}{ Awareness } \\
\hline 1961 Baumhart: HBR & 1700: HBR Subscribers & Industry climate influences ethical behavior. \\
\hline 1992 Tyson: JBE & 495: Students & $\begin{array}{l}\text { Individuals perceive their own behavior to be } \\
\text { more ethical than others. }\end{array}$ \\
\hline $\begin{array}{l}1992 \text { Dubinsky et al.: } \\
\text { JPSSM }\end{array}$ & 218: Salespeople & $\begin{array}{l}\text { Salespeople differ in their view of what is or is } \\
\text { not an ethical situation. }\end{array}$ \\
\hline $\begin{array}{l}1992 \text { Henthorne, Robin, } \\
\text { and Reidenbach: JBE }\end{array}$ & $\begin{array}{l}\text { 311: Sales managers } \\
\text { 329: Salespeople }\end{array}$ & $\begin{array}{l}\text { Managers have more critical view of questionable } \\
\text { behavior than salespeople. }\end{array}$ \\
\hline $\begin{array}{l}1993 \text { Kawathatzopoulos: } \\
\text { JBE }\end{array}$ & 31: Students & $\begin{array}{l}\text { Simple instruction is sufficient for a shift in } \\
\text { subject's mode of ethical problem solving. }\end{array}$ \\
\hline 1993 Morgan: AMR & 385: Managers & $\begin{array}{l}\text { Individuals perceive their own behavior to be } \\
\text { more ethical than others. }\end{array}$ \\
\hline $\begin{array}{l}1993 \text { O'Clock \& Okleshen: } \\
\text { JBE }\end{array}$ & 195: Students & $\begin{array}{l}\text { Individuals perceive their own behavior to be } \\
\text { more ethical than others. }\end{array}$ \\
\hline $\begin{array}{l}1993 \text { Robertson \& } \\
\text { Schlegelmilch: JBE }\end{array}$ & 813: Managers & $\begin{array}{l}\text { U.S. managers consider most ethical issues to be } \\
\text { more important than U.K. managers; U.S. } \\
\text { managers differ in their perception of the } \\
\text { importance of ethical issues. }\end{array}$ \\
\hline $\begin{array}{l}1993 \text { Shaub, Finn \& } \\
\text { Munter: BRA }\end{array}$ & 207: Audit CPAs & Ethical orientation influences ethical sensitivity. \\
\hline $\begin{array}{l}1993 \text { Stevens, Harris \& } \\
\text { Williamson: JBE }\end{array}$ & 171: Students and faculty & $\begin{array}{l}\text { Freshman and faculty differ in their awareness } \\
\text { levels in some instances. Seniors were more } \\
\text { ethically conservative than Freshman. }\end{array}$ \\
\hline $\begin{array}{l}1993 \text { White \& Dooley: } \\
\text { JBE }\end{array}$ & 184: Students & $\begin{array}{l}\text { Awareness of codes of conduct has no significant } \\
\text { impact on ethical decision or behavior. }\end{array}$ \\
\hline $\begin{array}{l}1994 \text { Kohut \& Corriher: } \\
\text { SAM AMJ }\end{array}$ & 86: MBA Students & $\begin{array}{l}\text { Awareness of codes of conduct has no significant } \\
\text { impact on ethical decision or behavior; no } \\
\text { significant difference in level of awareness by } \\
\text { position or level. }\end{array}$ \\
\hline $\begin{array}{l}1994 \text { Simpson, Banerjee } \\
\text { \& Simpson: JBE }\end{array}$ & 209: Students & $\begin{array}{l}\text { Awareness of codes of conduct has no significant } \\
\text { impact on ethical decision or behavior. }\end{array}$ \\
\hline 1996 Armstrong: JBE & $\begin{array}{l}\text { 197: Students } \\
\text { (3 different countries) }\end{array}$ & $\begin{array}{l}\text { Cultural environment influences the perception } \\
\text { of ethical situations. }\end{array}$ \\
\hline $\begin{array}{l}1996 \text { Wimalasiri, Pavri } \\
\text { \& Jalil: JBE }\end{array}$ & $\begin{array}{l}\text { 157: Managers and students } \\
\text { (Singapore) }\end{array}$ & $\begin{array}{l}\text { Gender and ethnicity is not significantly related } \\
\text { to ethical sensitivity; business managers and } \\
\text { students demonstrate the same ethical sensitivity. }\end{array}$ \\
\hline \multicolumn{3}{|c|}{ Individual factors: cognitive moral development } \\
\hline $\begin{array}{l}1990 \text { Trevino \& } \\
\text { Youngblood: JAP }\end{array}$ & 94: MBA Students & CMD influences ethical decision-making. \\
\hline 1992 Goolsby \& Hunt: JM & 269: AMA members & $\begin{array}{l}\text { CMD is positively related to socially responsible } \\
\text { behavior. }\end{array}$ \\
\hline
\end{tabular}


TABLE II (Continued)

\begin{tabular}{|c|c|c|}
\hline Yr./Author: Journal & N: Sample composition & Findings \\
\hline 1993 Elm \& Nichols: JBE & 243: Managers & $\begin{array}{l}\text { Age and education level are negatively related } \\
\text { CMD. }\end{array}$ \\
\hline 1990 Weber: HR & 37: Managers & $\begin{array}{l}\text { Level of moral reasoning in a business context is } \\
\text { lower than in nonbusiness. }\end{array}$ \\
\hline $\begin{array}{l}1996 \text { Wimalasiri, Pavri \& } \\
\text { Jalil: JBE }\end{array}$ & $\begin{array}{l}\text { 109: Managers } \\
\text { 48: Business students }\end{array}$ & $\begin{array}{l}\text { Age, education and religious affiliation influences } \\
\text { CMD. }\end{array}$ \\
\hline $\begin{array}{l}1993 \text { Sridhar \& Camburn: } \\
\text { JBE }\end{array}$ & 246: Students & $\begin{array}{l}\text { Organizations develop along similar stages and } \\
\text { levels as individuals. }\end{array}$ \\
\hline \multicolumn{3}{|c|}{ Individual factors: moral philosophy } \\
\hline 1978 Hegarty \& Sims: JAP & 120: Graduate students & $\begin{array}{l}\text { Machiavellianism is a significant covariant of } \\
\text { unethical behavior. }\end{array}$ \\
\hline $\begin{array}{l}1989 \text { Swinyard, DeLong, } \\
\text { \& Cheng: JBE }\end{array}$ & $\begin{array}{l}\text { 568: Students } \\
\text { (U.S. and Chinese) }\end{array}$ & $\begin{array}{l}\text { U.S. students base ethical behavior more on } \\
\text { teleological philosophy; students are equivalent in } \\
\text { their moral acceptance of a decision. }\end{array}$ \\
\hline $\begin{array}{l}1990 \text { Mayo \& Marks: } \\
\text { JAMS }\end{array}$ & 104: Marketing researchers & $\begin{array}{l}\text { Deontological and teleological philosophies have } \\
\text { a significant influence on ethical judgments. }\end{array}$ \\
\hline $\begin{array}{l}1992 \text { Fraedrich \& Ferrell: } \\
\text { JAMS }\end{array}$ & 184: Marketing managers & $\begin{array}{l}\text { Managers change moral philosophy based on the } \\
\text { situation. }\end{array}$ \\
\hline 1993 Allen \& Davis: JBE & 207: Consultants & $\begin{array}{l}\text { Individual values are positively related to } \\
\text { professional ethics. }\end{array}$ \\
\hline $\begin{array}{l}1993 \text { Cohen, Pant \& } \\
\text { Sharp: JBE }\end{array}$ & 113: Accounting academics & Accountants use different philosophies. \\
\hline 1993 Fraedrich: JBE & 189: Managers & $\begin{array}{l}\text { Rule deontologists rank higher on ethical behavior } \\
\text { scale than any other philosophy types. }\end{array}$ \\
\hline $\begin{array}{l}1993 \text { Galbraith \& } \\
\text { Stephenson: JBE }\end{array}$ & 107: Students & $\begin{array}{l}\text { Type of situation leads to different decision criteria } \\
\text { for males vs. females; neither males nor females } \\
\text { use one decision making criteria. }\end{array}$ \\
\hline $\begin{array}{l}1993 \text { Glenn \& Van Loo: } \\
\text { JBE }\end{array}$ & 1668: Students & $\begin{array}{l}\text { Students are less ethical than managers and have } \\
\text { lower ethical attitudes. }\end{array}$ \\
\hline $\begin{array}{l}1993 \text { Hunt \& Vasquez- } \\
\text { Parraga: JMR }\end{array}$ & 747: Managers & $\begin{array}{l}\text { Deontological unethical behavior with negative } \\
\text { consequences is disciplined more severely and is } \\
\text { rewarded more with positive consequences. }\end{array}$ \\
\hline $\begin{array}{l}1993 \text { Singhapakdi \& Vitell: } \\
\text { JBE }\end{array}$ & 492: AMA members & $\begin{array}{l}\text { Professional values and certain personal values are } \\
\text { factors in ethical judgments. }\end{array}$ \\
\hline $\begin{array}{l}1993 \text { Vitell, Rallapalli \& } \\
\text { Singhapakdi: JAMS }\end{array}$ & 508: AMA Members & $\begin{array}{l}\text { Ethical climate has no significant effect upon } \\
\text { personal norms; relativism is a poor predictor of } \\
\text { norms; established } 5 \text { dimensions of ethical } \\
\text { marketing norms. }\end{array}$ \\
\hline $\begin{array}{l}1993 \text { Zabid \& Alsogoff: } \\
\text { JBE }\end{array}$ & 81: Malaysian managers & Malaysian managers have high ethical values. \\
\hline $\begin{array}{l}1994 \text { Cyriac \& Dharmaraj: } \\
\text { JBE }\end{array}$ & $\begin{array}{l}\text { 68: Middle and senior } \\
\text { level managers }\end{array}$ & Machiavellianism influences ethical decisions. \\
\hline
\end{tabular}


TABLE II (Continued)

\begin{tabular}{|c|c|c|}
\hline Yr./Author: Journal & N: Sample composition & Findings \\
\hline 1994 Grover \& Hui: JBE & 248: Students & $\begin{array}{l}\text { Individuals change philosophies based upon the } \\
\text { situation. }\end{array}$ \\
\hline 1994 Tansey et al.: JPSSM & 104: Sales people & Moral philosophy affects ethical decision. \\
\hline $\begin{array}{l}1996 \text { Brady \& Wheeler: } \\
\text { JBE }\end{array}$ & $\begin{array}{l}\text { 141: Financial institution } \\
\text { employees }\end{array}$ & $\begin{array}{l}\text { Ethical philosophy is more behavioral-oriented } \\
\text { (people tend to behave before they think). }\end{array}$ \\
\hline 1996 LaFleur et al.: JAMS & $\begin{array}{l}\text { 251: Advertising } \\
\text { practitioners }\end{array}$ & $\begin{array}{l}\text { Rule configuration (moral philosophy) influences } \\
\text { ethical intentions and judgment. }\end{array}$ \\
\hline $\begin{array}{l}1996 \text { McDonald \& Pak: } \\
\text { JBE }\end{array}$ & $\begin{array}{l}\text { 4044: MBA students } \\
\text { employed full time; business } \\
\text { association members }\end{array}$ & $\begin{array}{l}\text { Multiple cognitive frameworks (philosophies) are } \\
\text { used to evaluate ethical content of marketing } \\
\text { activities. }\end{array}$ \\
\hline $\begin{array}{l}1996 \text { Verbeke, Ouwerkerk } \\
\text { \& Peelen: JBE }\end{array}$ & 185: Salespeople & $\begin{array}{l}\text { Personality traits (Machiavellianism) affects ethical } \\
\text { decision-making. }\end{array}$ \\
\hline 1997 Akaah: JBR & 452: AMA members & $\begin{array}{l}\text { People form research ethics judgments based } \\
\text { primarily on deontological considerations } \\
\text { secondarily on teleological considerations. }\end{array}$ \\
\hline \multicolumn{3}{|c|}{ Individual factors: gender } \\
\hline 1978 Hegarty \& Sims: JAP & 120: Graduate Students & No significant gender differences. \\
\hline $\begin{array}{l}1983 \text { Browning \& } \\
\text { Zabriskie: IMM }\end{array}$ & 145: Purchasing association & No significant gender differences. \\
\hline $\begin{array}{l}1984 \text { Beltramini, Peterson } \\
\text { \& Kozmetsky: JBE }\end{array}$ & 2856: Students & Females act more concerned with ethical issues. \\
\hline $\begin{array}{l}1985 \text { Chonko \& Hunt: } \\
\text { JBR }\end{array}$ & 1076: Managers & $\begin{array}{l}\text { Males acknowledged fewer ethical problems than } \\
\text { females. }\end{array}$ \\
\hline $\begin{array}{l}1985 \text { Dubinsky \& Levy: } \\
\text { JAMS }\end{array}$ & 122: Retail sales people & No significant gender differences. \\
\hline $\begin{array}{l}1985 \text { McNichols \& } \\
\text { Zimmerer: JBE }\end{array}$ & 1178: Students & No significant gender differences. \\
\hline $\begin{array}{l}1987 \text { Kidwell, Stevens \& } \\
\text { Bethke: JBE }\end{array}$ & 100: Managers & $\begin{array}{l}\text { Males amd females differ slightly; males are more } \\
\text { likely to hide their mistakes. }\end{array}$ \\
\hline $\begin{array}{l}1988 \text { Ferrell \& Skinner: } \\
\text { JMR }\end{array}$ & 602: Marketing researchers & $\begin{array}{l}\text { Gender is a significant predictor of ethical } \\
\text { behavior. }\end{array}$ \\
\hline $\begin{array}{l}1988 \text { Jones \& Gautschi: } \\
\text { JBE }\end{array}$ & 455: MBA Students & $\begin{array}{l}\text { Females are less likely than males to be loyal to } \\
\text { the organization in an ethically questionable } \\
\text { environment. }\end{array}$ \\
\hline 1989 Akaah: JBE & 420: AMA Members & $\begin{array}{l}\text { Females indicate higher ethical judgment than } \\
\text { males. }\end{array}$ \\
\hline $\begin{array}{l}1989 \text { Barnett \& Karson: } \\
\text { JBE }\end{array}$ & $\begin{array}{l}\text { 513: Insurance company } \\
\text { employees }\end{array}$ & Females more ethical than males. \\
\hline 1989 Bellizi \& Hite: JM & $\begin{array}{l}\text { 452: Sales managers and } \\
\text { executives }\end{array}$ & Gender influences ethical behavior. \\
\hline $\begin{array}{l}1989 \text { Betz, O'Connell } \\
\text { \& Shepherd: JBE }\end{array}$ & 213: Students & $\begin{array}{l}\text { Males more than twice as likely as females to } \\
\text { engage in unethical behavior. }\end{array}$ \\
\hline
\end{tabular}


TABLE II (Continued)

\begin{tabular}{|c|c|c|c|}
\hline Yr./A & Author: Journal & N: Sample composition & Findings \\
\hline 1989 & 9 Derry: JBE & $\begin{array}{l}\text { 40: Fortune } 100 \text { industrial } \\
\text { corporate employees }\end{array}$ & $\begin{array}{l}\text { Gender not related to reported experience of } \\
\text { moral conflict }\end{array}$ \\
\hline 1990 & $\begin{array}{l}0 \text { Kelley, Ferrell \& } \\
\text { Skinner: JBE }\end{array}$ & $\begin{array}{l}\text { 550: Marketing researchers } \\
\text { (AMA) }\end{array}$ & Females more ethical than males. \\
\hline 1990 & $\begin{array}{l}0 \text { Singhapakdi \& } \\
\text { Vitell: JMM }\end{array}$ & 529: AMA Members & No significant gender differences. \\
\hline 1992 & $\begin{array}{l}2 \text { Borkowski \& Ugras: } \\
\text { JBE }\end{array}$ & $\begin{array}{l}\text { 90: Students } \\
\text { 40: MBA students }\end{array}$ & $\begin{array}{l}\text { Males and MBAs are more utilitarian; } \\
\text { females have more definite ethical positions. }\end{array}$ \\
\hline 1992 & 2 Callan: JBE & 2261: State employees & No significant gender differences. \\
\hline 1992 & 2 Dawson: JPSSM & 89: Students & $\begin{array}{l}\text { Females operate with higher standard of ethical } \\
\text { behavior than males. }\end{array}$ \\
\hline 1992 & $\begin{array}{l}2 \text { Goolsby \& Hunt: } \\
\text { JM }\end{array}$ & 269: AMA members & $\begin{array}{l}\text { High CME profile more likely to be female and } \\
\text { highly educated. }\end{array}$ \\
\hline 1992 & 2 Ruegger \& King: JBE & 2196: Students & Females more ethical than males. \\
\hline 1992 & 2 Serwinek: JBE & 423: Insurance employees & No significant differences in majority of indices. \\
\hline 1992 & 2 Tyson: JBE & $\begin{array}{l}\text { 495: Students and } \\
\text { accountants }\end{array}$ & Females have greater ethical sensitivity. \\
\hline 1992 & $\begin{array}{l}2 \text { Whipple \& Swords: } \\
\text { JBE }\end{array}$ & 319: Students & $\begin{array}{l}\text { Females are more critical of ethical issues than } \\
\text { males. }\end{array}$ \\
\hline 1993 & $\begin{array}{l}3 \text { Galbraith \& } \\
\text { Stephenson: JBE }\end{array}$ & 107: Students & $\begin{array}{l}\text { Genders differ in use of decision approach when } \\
\text { making ethical judgments. }\end{array}$ \\
\hline 1996 & $\begin{array}{l}6 \text { Brady \& Wheeler: } \\
\text { JBE }\end{array}$ & $\begin{array}{l}\text { 14: Financial institution } \\
\text { employees }\end{array}$ & $\begin{array}{l}\text { Gender is not a significant indicator of ethical } \\
\text { disposition. }\end{array}$ \\
\hline \multicolumn{4}{|c|}{ Individual factors: age } \\
\hline 1983 & $\begin{array}{l}3 \text { Browning \& } \\
\text { Zabriskie: IMM }\end{array}$ & $\begin{array}{l}\text { 145: Purchasing association } \\
\text { members }\end{array}$ & $\begin{array}{l}\text { Younger managers have a more ethical viewpoint } \\
\text { than older managers. }\end{array}$ \\
\hline 1984 & 4 Stevens: ABER & $\begin{array}{l}\text { 349: Students } \\
\text { 113: Executives }\end{array}$ & No significant age differences. \\
\hline 1987 & $\begin{array}{l}7 \text { Kidwell, Stevens \& } \\
\text { Bethke: JBE }\end{array}$ & 100: Managers & No significant age differences. \\
\hline 1988 & 8 Izraeli: JBE & 97: Managers & No significant age differences. \\
\hline 1988 & 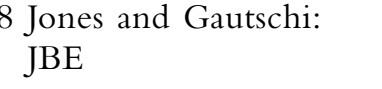 & 455: MBA Students & Minimal significance on 2 of 14 items. \\
\hline 1989 & $\begin{array}{l}9 \text { Barnett \& Karson: } \\
\text { JBE }\end{array}$ & $\begin{array}{l}\text { 513: Insurance company } \\
\text { employees }\end{array}$ & Later career stages generally more ethical. \\
\hline & $\begin{array}{l}0 \text { Kelley, Ferrell \& } \\
\text { Skinner: JBE }\end{array}$ & $\begin{array}{l}\text { 550: Marketing researchers } \\
\text { (AMA) }\end{array}$ & $\begin{array}{l}\text { Older respondents and those with greater than } \\
10 \text { years experience are more ethical. }\end{array}$ \\
\hline 1992 & 2 Muncy \& Vitell: JBR & 569: Consumers & $\begin{array}{l}\text { Individuals with greater ethical concern are older, } \\
\text { have lower income and less education. }\end{array}$ \\
\hline 1992 & 2 Ruegger \& King: JBE & 2196: Students & Older students are more ethical. \\
\hline 1992 & 2 Serwinek: JBE & 423: Insurance employees & $\begin{array}{l}\text { Older workers have stricter interpretations of } \\
\text { ethical standards. }\end{array}$ \\
\hline
\end{tabular}


TABLE II (Continued)

\begin{tabular}{|c|c|c|}
\hline Yr./Author: Journal & N: Sample composition & Findings \\
\hline 1992 Tyson: JBE & $\begin{array}{l}\text { 495: Students and } \\
\text { accountants }\end{array}$ & $\begin{array}{l}\text { Regardless of age, individuals rated themselves as } \\
\text { more ethical than others. }\end{array}$ \\
\hline $\begin{array}{l}1993 \text { Stevens, Harris \& } \\
\text { Williamson: JBE }\end{array}$ & $\begin{array}{l}\text { 171: Students and } \\
\text { professors }\end{array}$ & $\begin{array}{l}\text { Seniors are more ethically conservative than } \\
\text { freshmen. }\end{array}$ \\
\hline $\begin{array}{l}1993 \text { White \& Dooley: } \\
\text { JBE }\end{array}$ & 184: Students & Students respond more practically than ethically. \\
\hline $\begin{array}{l}1994 \text { Kohut \& Corriher: } \\
\text { SAM AMJ }\end{array}$ & 86: MBA Students & No significant age differences. \\
\hline $\begin{array}{l}1996 \text { Brady \& Wheeler: } \\
\text { JBE }\end{array}$ & $\begin{array}{l}\text { 141: Financial institution } \\
\text { employees }\end{array}$ & $\begin{array}{l}\text { Age is a powerful determinant regarding ethical } \\
\text { disposition. }\end{array}$ \\
\hline \multicolumn{3}{|c|}{ Individual factors: education and work experience } \\
\hline $\begin{array}{l}1972 \text { Hawkins \& } \\
\text { Cocanougher: JM }\end{array}$ & 225: Students & $\begin{array}{l}\text { Business majors tend to be more tolerant of } \\
\text { questionable business practices. }\end{array}$ \\
\hline $\begin{array}{l}1974 \text { Goodman \& } \\
\text { Crawford: PJ }\end{array}$ & 1500: Students & No significant differences found. \\
\hline $\begin{array}{l}1980 \text { Arlow \& Ulrich: } \\
\text { ABER }\end{array}$ & $\begin{array}{l}\text { 120: Students } \\
\text { 103: Executives }\end{array}$ & Executives are more ethical than students. \\
\hline $\begin{array}{l}1981 \text { Dubinsky \& Gwin: } \\
\text { JPMM }\end{array}$ & $\begin{array}{l}\text { 226: Sales and purchasing } \\
\text { managers }\end{array}$ & $\begin{array}{l}\text { Purchasing managers see more questionable } \\
\text { business practices than sales managers. }\end{array}$ \\
\hline $\begin{array}{l}1983 \text { Browning \& } \\
\text { Zabriskie: IMM }\end{array}$ & 145: Purchasing association & $\begin{array}{l}\text { Managers with higher level of education viewed } \\
\text { gifts as unethical. }\end{array}$ \\
\hline $\begin{array}{l}1984 \text { Beltramini, Peterson } \\
\text { \& Kozmetsky: JBE }\end{array}$ & 2856: Students & $\begin{array}{l}\text { Business majors are more concerned with ethical } \\
\text { issues than other majors. }\end{array}$ \\
\hline $\begin{array}{l}1984 \text { Dubinsky \& Ingram: } \\
\text { JBE }\end{array}$ & 116: Salespeople & No significant differences. \\
\hline 1984 Stevens: JBR & $\begin{array}{l}\text { 349: Students } \\
\text { 113: Executives }\end{array}$ & Executives more ethical than students. \\
\hline $\begin{array}{l}1985 \text { Chonko \& Hunt: } \\
\text { JBR }\end{array}$ & 1076: Managers & $\begin{array}{l}\text { Technical majors tend to be more ethical than } \\
\text { nontechnical majors. }\end{array}$ \\
\hline $\begin{array}{l}1985 \text { McNichols \& } \\
\text { Zimmerer: JBE }\end{array}$ & 1178: Students & No significant differences. \\
\hline $\begin{array}{l}1987 \text { Kidwell, Stevens \& } \\
\text { Bethke: JBE }\end{array}$ & 100: Managers & $\begin{array}{l}\text { The greater the work experience, the more ethical } \\
\text { the responses. }\end{array}$ \\
\hline $\begin{array}{l}1987 \text { Laczniak \& } \\
\text { Inderrieden: JBE }\end{array}$ & 113: MBA students & $\begin{array}{l}\text { Education (technical versus nontechnical) has no } \\
\text { effect. }\end{array}$ \\
\hline $\begin{array}{l}1988 \text { Lane, Schaupp \& } \\
\text { Parsons: JBE }\end{array}$ & $\begin{array}{l}\text { 335: Graduate and } \\
\text { Undergraduate students }\end{array}$ & Minimal significant differences. \\
\hline $\begin{array}{l}1989 \text { Stevens, Richardson } \\
\text { \& Abramowitz: } \\
\text { AMAP }\end{array}$ & $\begin{array}{l}\text { 382: Students, managers } \\
\text { and Attorneys }\end{array}$ & No significant differences. \\
\hline 1992 Callan: JBE & 226: State employees & $\begin{array}{l}\text { Length of employment not related to ethical } \\
\text { values. }\end{array}$ \\
\hline
\end{tabular}


TABLE II (Continued)

\begin{tabular}{|c|c|c|}
\hline Yr./Author: Journal & N: Sample composition & Findings \\
\hline $\begin{array}{l}1992 \text { Henthorne, Robin } \\
\text { \& Reidenbach: JBE }\end{array}$ & $\begin{array}{l}\text { 311: Sales mangers } \\
\text { 329: Salespeople }\end{array}$ & $\begin{array}{l}\text { Substantial variance between the responses from } \\
\text { retail managers vs. retail salespeople; } \\
\text { managers viewed the scenarios more critically } \\
\text { than salespeople. }\end{array}$ \\
\hline 1992 Serwinek: JBE & 423: Insurance employees & No significant differences. \\
\hline $\begin{array}{l}1994 \text { Kohut \& Corriher: } \\
\text { SAM AMJ }\end{array}$ & 86: MBA Students & $\begin{array}{l}\text { No relationship between experience or position } \\
\text { and ethical decision-making. }\end{array}$ \\
\hline
\end{tabular}

Individual factors: nationality

1978 Hegarty \& Sims: JAP 120: Graduate students

1987 Becker \& Fritzsche: $\quad$ 72: French; $\mathrm{JBE}$

1992 Abratt, Nel \& Higgs: JBE

1992 Kaye: JBE

1992 Small: JBE

1992 Whipple \& Swords: JBE

1992 White \& Rhodeback: JBE

1993 Alam: JBE

1993 Robertson \& Schlegelmilch: JBE

1993 Zabid \& Alsagoff: JBE
Foreign students are more ethical than U.S. students.

French managers have strongest beliefs in codes of ethics.
124: U.S. managers

52: South African and Australian managers

50: Australian and U.S. companies

179: Students

319: U.S. and U.K. students

118: Graduate students 267: Taiwanese managers

99: Top 200 New Zealand companies

813: U.S. managers

860: U.K. managers

81: Malaysian managers
South African and Australian managers show no significant differences in responses.

U.S. firms have more formal ethical structures than Australian firms.

U.S. and Australian students have similar attitudes; U.S. students slightly stronger ethical sensitivity.

No significant differences.

U.S. managers have a higher ethical rating than Taiwanese managers.

New Zealand CEOs give low priority to ethical values.

U.S. managers consider ethical issues to be more important than U.K. managers.

Malaysian managers have relatively high ethical values.

\section{Hegarty \& Sims: JAP \\ 1985 McNichols \& Zimmerer: JBE \\ 1987 Kidwell, Stevens \& Bethke: JBE}

\section{Individual factors: religion}

120: Graduate students

1178: Students

100: Managers
No significant findings.

Strong religious beliefs related to a negative attitude toward certain acceptable behaviors. No significant findings.

\section{Individual factors: locus of control}

1978 Hegarty \& Sims: JAP

1989 Zahra: JBE
120: Graduate students

302: Managers
Locus of control is not related to ethical decisionmaking.

Managers with an external locus of control perceived organizational politics as ethical. 
TABLE II (Continued)

\begin{tabular}{|c|c|c|}
\hline Yr./Author: Journal & N: Sample composition & Findings \\
\hline $\begin{array}{l}1990 \text { Klebe-Trevino \& } \\
\text { Youngblood: JAP }\end{array}$ & 94: MBA students & $\begin{array}{l}\text { Locus of control influences ethical decision- } \\
\text { making directly and indirectly through outcome } \\
\text { expectancies. }\end{array}$ \\
\hline $\begin{array}{l}1990 \text { Singhapakdi \& Vitell: } \\
\text { JMM }\end{array}$ & 529: AMA members & No significant findings. \\
\hline \multicolumn{3}{|c|}{ Individual factors: intent } \\
\hline $\begin{array}{l}1989 \text { Dubinsky \& Loken: } \\
\text { JBR }\end{array}$ & 305: Salespeople & $\begin{array}{l}\text { Subjective norms and attitudes are good predictors } \\
\text { of intentions. }\end{array}$ \\
\hline $\begin{array}{l}1990 \text { Mayo \& Marks: } \\
\text { JAMS }\end{array}$ & 104: Marketing researchers & $\begin{array}{l}\text { Deontological and Teleological evaluations have a } \\
\text { significant influence on ethical intent. }\end{array}$ \\
\hline $\begin{array}{l}\text { 1990, Reidenbach \& } \\
\text { Robin: SMA }\end{array}$ & 103: Sales personnel & $\begin{array}{l}\text { Ethical attitudes are strongly linked to ethical } \\
\text { intentions. }\end{array}$ \\
\hline $\begin{array}{l}1996 \text { Robin, Reidenbach, } \\
\text { \& Forrest: JBR }\end{array}$ & 251: Advertisers & $\begin{array}{l}\text { Perceived importance of ethical issue influences } \\
\text { behavioral intention. }\end{array}$ \\
\hline \multicolumn{3}{|c|}{ Individual factors: moral intensity } \\
\hline $\begin{array}{l}1996 \text { Robin, Reidenbach, } \\
\text { \& Forrest: JBR }\end{array}$ & 251: Advertisers & $\begin{array}{l}\text { Perceived importance of ethical issue influences } \\
\text { behavioral intention. }\end{array}$ \\
\hline $\begin{array}{l}1996 \text { Singhapakdi, Vitell } \\
\text { \& Kraft: JBR }\end{array}$ & 453: AMA members & $\begin{array}{l}\text { Moral intensity influences the ethical decision- } \\
\text { making process. }\end{array}$ \\
\hline
\end{tabular}

the culture of the organization contributes to managing organizational ethics.

\section{Codes of ethics}

The structural elements of an ethics and compliance program contribute to ethical decision making in organizations following the importance of the organizational culture and climate. Key in this understanding is the articulation of the organizational risk areas and the values of top management expressed through the code of ethics (or conduct). Seventeen studies address the role of codes of ethics in influencing organizational ethical decision making. A majority of the studies revealed that codes influence ethical decision making and assist in raising the general level of awareness of ethical issues. The passage of the Federal Sentencing Guidelines for Organizations in 1991, which outlined seven fundamental steps to developing effective com- pliance programs, spotlighted codes of ethics as key to effectuating higher levels of ethical climate. Perhaps our greatest opportunities for research relate to evaluating the effectiveness of codes, structuring of codes, and their communication and integration with other aspects of the organization's culture.

\section{E. Awareness}

Fifteen studies address awareness of ethical issues. A majority of these studies utilized student samples (nine). The findings were widely diverse based upon the goals of the studies. Several evaluate the role of codes of conduct in generating awareness of ethical issues (Kohut and Corriher, 1994; Simpson, Banerjee and Simpson, 1994; White and Dooley, 1993). Others consider individuals' awareness of their own ethical behavior relative to others' (Morgan, 1993; O'Clock and Okleshen, 1993; Tyson, 1992), 
TABLE III

Moderating factors of ethical decision-making in business

\begin{tabular}{|c|c|c|}
\hline Yr./Author: Journal & N: Sample composition & Findings \\
\hline \multicolumn{3}{|c|}{ Organizational factors: opportunity } \\
\hline $\begin{array}{l}1979 \text { Zey-Ferrell, Weaver } \\
\text { \& Ferrell: HR }\end{array}$ & $\begin{array}{l}\text { 133: AMA marketing } \\
\text { managers }\end{array}$ & $\begin{array}{l}\text { Opportunity and significant others are better } \\
\text { indicators of ethical behavior than personal factors. }\end{array}$ \\
\hline $\begin{array}{l}1982 \text { Zey-Ferrell \& } \\
\text { Ferrell: HR }\end{array}$ & 225: Advertising managers & Opportunity is a predictor of ethical behavior. \\
\hline 1993 Wahn: JBE & $\begin{array}{l}\text { 565: Human resource } \\
\text { professionals }\end{array}$ & $\begin{array}{l}\text { The greater the organizational dependence, the } \\
\text { more likely the compliance with organizational } \\
\text { pressure to behave unethically. }\end{array}$ \\
\hline
\end{tabular}

1977 Brenner \& Molander: HBR

1977 Weaver \& Ferrell: AMAP

1978 Ferrell \& Weaver: JM

1990 Trevino \& Youngblood: JAP

1992 Barnett: JBE

1992 Dubinsky et al.: JPSSM

1992 Kaye: JBE

1993 Allen and Davis: JBE

1993 Barnett, Cochran \& Taylor: JBE

1993 Beneish \& Chatov: JAPP

1993 Glenn \& Van Loo: JBE

1993 Kawathatzopoulos: JBE

1993 Robertson \& Schlegelmilch: JBE

1994 Bruce: PPMR

Organizational factors: opportunity; codes of ethics

1227: HBR subscribers

280: AMA members

236: Marketing managers

94: MBA students

240: Business executives

218: Salespeople

50: Australian companies

207: Consultants

295: Business executives

160: Managers

1668: Students

31: Students

813: Managers

522: Managers
Codes are second to superior behavior in influencing ethical behavior.

Codes and enforcement improve ethical behavior.

Existence/enforcement of a corporate policy does not support more ethical conduct.

Formal policies generate an increased level of awareness and subsequent reporting of unethical incidents.

Formal policies generate an increased level of awareness and subsequent reporting of unethical incidents.

Employees desire more direction through formal policies and codes of ethics.

Formal policies generate an increased level of awareness and subsequent reporting of unethical incidents.

Codes must be enforced to be effective.

Formal policies generate an increased level of awareness and subsequent reporting of unethical incidents.

Contents of codes vary according to industry.

Codes are less effective than earlier research indicated.

Simple instruction is sufficient for a significant shift in the subject's mode of ethical problem solving.

U.K. companies' communicate policies through senior executives and U.S. communicate policies through the HR department.

Codes are necessary, but not as effective as example, support and education. 
TABLE III (Continued)

\begin{tabular}{|c|c|c|}
\hline Yr./Author: Journal & N: Sample composition & Findings \\
\hline $\begin{array}{l}1994 \text { Kohut \& Corriher: } \\
\text { SAM AMJ }\end{array}$ & 86: MBA students & $\begin{array}{l}\text { Knowledge of code has no significant impact on } \\
\text { ethical decisions. }\end{array}$ \\
\hline $\begin{array}{l}1996 \text { McCabe, Trevino \& } \\
\text { Butterfield: BEQ }\end{array}$ & $\begin{array}{l}\text { 318: People employed in } \\
\text { business }\end{array}$ & $\begin{array}{l}\text { Codes of conduct are positively associated with } \\
\text { ethical behavior. }\end{array}$ \\
\hline $\begin{array}{l}1996 \text { Verbeke, Ouwerkerk } \\
\text { \& Peelen: JBE }\end{array}$ & 185: Salespeople & $\begin{array}{l}\text { Internal communication and choice of control } \\
\text { system affects ethical decision-making. }\end{array}$ \\
\hline
\end{tabular}

Organizational factors: opportunity; rewards and sanctions

1961 Baumhart: HBR

1700: HBR subscribers

1978 Hegarty \& Sims: JAP

1989 Bellizi \& Hite: JM

1990 Hunt, Kiecker \& Chonko: JAMS

1990 Trevino \& Youngblood: JAP

1992 Barnett: JBE

1992 Callahan \& Collins: JBE

1992 Dabholkar \& Kellaris: JBR

1992 DeConinck: JBE

1992 Trevino \& Victor: AMJ

1993 Barnett, Cochran \&

1993 Hunt \& VasquezParraga: JMR

1993 Schultz et al.: JAR
246: Sales managers

120: Graduate students

452: Managers and executives

330: Advertising executives

94: MBA students

240: Executives

276: NY State employees

198: Sales managers

478: Students

295: Executives

747: Managers

145: Managers
Rewards for ethical behavior increases ethical behavior.

Rewards for unethical behavior increases its frequency.

Poor performers are disciplined more harshly when caught in questionable activity than good performers; when negative consequences result from the behavior there is harsher punishment.

Neither penalties nor rewards are associated with socially responsible actions.

Rewards influence ethical decision-making indirectly through outcome expectations.

Executives report increased frequency of employee-voiced concerns in larger or nonunionized organizations; unionized companies perceive higher levels of whistleblowing.

Belief that there is an informal hierarchy for whistleblowing; fear of reprisal deters whistleblowing.

Controversial sales practices with direct $\$$ consequences and practices involving transgressions against customers are judged most severely.

Consequences of unethical behavior reflect the strength of the discipline received with poor performers receiving the harshest discipline.

Unethical behavior tends to be reported by those with responsibility and when others are perceived to be hurt.

Policies encouraging disclosure are positively associated with increased reporting; ethics policies are positively associated with greater disclosure.

Unethical behavior is disciplined more severely when results are negative and rewarded for positive results.

Organizational prosperity is not related to reporting, but the situation relates positively to reporting. 
TABLE III (Continued)

\begin{tabular}{lll}
\hline Yr./Author: Journal & N: Sample composition & \multicolumn{1}{c}{ Findings } \\
\hline $\begin{array}{l}1993 \text { Victor, Trevino \& } \\
\text { Shapiro: JBE }\end{array}$ & 159: Fast food employees & $\begin{array}{l}\text { Inclination to report peers is positively associated } \\
\text { with role perception, interest of group members } \\
\text { and procedural justice. }\end{array}$ \\
1997 Akaah: JBR & 452: AMA members & $\begin{array}{l}\text { Assessment of the appropriateness of reward/ } \\
\text { discipline for ethical unethical research behavior } \\
\text { is guided solely by ethical judgments in the case } \\
\text { of reward, and partly by ethical judgments and } \\
\text { partly by teleological considerations in case of } \\
\text { discipline. }\end{array}$ \\
\hline
\end{tabular}

Organizational factors: culture and climate

1961 Baumhart: HBR

1977 Weaver \& Ferrell: AMAP

1988 Ferrell \& Skinner: JMR

1988 Victor \& Cullen: ASQ

1992 Dabholkar \& Kellaris: JBR

1992 DeConinck: JBE

1992 Kaye: JBE

1992 Small: JBE

1992 Wang \& Coffey: JBE

1993 Alam: JBE

1993 Elm \& Nichols: JBE

1993 Posner \& Schmidt: JBE

1993 Vitell, Rallapalli \& Singhapakdi: JAMS

1994 Judge: JBE

1994 Soutar, McNeil \& Molster: JBE

1995 Jones \& Hiltebeitel: JBE
1700: HBR subscribers

280: AMA members

602: Marketing researchers

872: Managers

198: Sales managers

246: Sales managers

50: Australian companies

179: Students

78: Fortune 500 Board of Directors

99: New Zealand companies

243: Managers

1059: Managers

508: AMA members

162: Hospitals

105: Western Australian managers

250: Members of the institute of management accountants
Industry climate influences ethical decisionmaking.

Existence and enforcement of a corporate policy on ethics may improve some ethical beliefs.

Organizational structure or bureaucracy is related to ethical behavior.

Identifies 5 dimensions in ethical work climate.

Organizational controversial practices which are targeted to customers are judged most severely.

Poor performers receive the harshest discipline for unethical behavior.

U.S. firms have more cultural support of ethics throughout the organization than Australian firms.

Similarities exist between U.S. and Australian attitudes toward business ethics.

Social responsibility receives greater support when more outsiders are on the board.

New Zealand companies give low priority to ethical values within the organization.

Ethical climate is unrelated to moral reasoning.

Supports values congruence model.

Support such as a code of ethics contributes to ethical climate.

Organizational size and economic scarcity are negatively related to the social performance of the organization.

Organizational factors have a significant impact on the ethicalness of the firm.

Organizational support influences ethical decision processes. 
TABLE III (Continued)

\begin{tabular}{|c|c|c|}
\hline Yr./Author: Journal & N: Sample composition & Findings \\
\hline $\begin{array}{l}1997 \text { Schwepker, Ferrell } \\
\text { \& Ingram: JAMS }\end{array}$ & $\begin{array}{l}\text { 152: Employees of Sales } \\
\text { and marketing executive } \\
\text { member firms }\end{array}$ & $\begin{array}{l}\text { Ethical climate is negatively associated with } \\
\text { perceived ethical conflict. }\end{array}$ \\
\hline $\begin{array}{l}1996 \text { Verbeke, Ouwerkerk } \\
\text { \& Peelen: JBE }\end{array}$ & 185: Salespeople & Ethical climate affects ethical decision-making. \\
\hline \multicolumn{3}{|c|}{ Organizational factors: significant others } \\
\hline 1961 Baumhart: HBR & 1700: HBR subscribers & $\begin{array}{l}\text { Significant others influence ethical decision- } \\
\text { making. }\end{array}$ \\
\hline $\begin{array}{l}1977 \text { Brenner \& Molander } \\
\text { (77): HBR }\end{array}$ & 1227: HBR subscribers & $\begin{array}{l}\text { Significant others influence ethical behavior; codes } \\
\text { second to supervisor behavior. }\end{array}$ \\
\hline $\begin{array}{l}1979 \text { Zey-Ferrell, Weaver } \\
\text { \& Ferrell: HR }\end{array}$ & $\begin{array}{l}\text { 133: AMA marketing } \\
\text { managers }\end{array}$ & $\begin{array}{l}\text { Significant others and opportunity better } \\
\text { indicators of ethical behavior. }\end{array}$ \\
\hline $\begin{array}{l}1982 \text { Zey-Ferrell \& } \\
\text { Ferrell: HR }\end{array}$ & $\begin{array}{l}\text { 225: Managers and ad } \\
\text { agency managers }\end{array}$ & $\begin{array}{l}\text { Significant others predictor of ethical behavior; } \\
\text { significant others best predictor of ethical behavior. }\end{array}$ \\
\hline 1992 Tyson: JBE & 495: Students & $\begin{array}{l}\text { A relationship exists between significant others and } \\
\text { ethical decision-making in the organization. }\end{array}$ \\
\hline 1993 Morgan: AMJ & 385: Managers & $\begin{array}{l}\text { Perception of manager varied according to } \\
\text { perspective of rater; ethical behavior enhances } \\
\text { managers' stature in eyes of subordinates. }\end{array}$ \\
\hline 1993 Wahn: JBE & $\begin{array}{l}\text { 565: Human Resource } \\
\text { Professionals }\end{array}$ & $\begin{array}{l}\text { A relationship exists between significant others and } \\
\text { ethical decision-making in the organization. }\end{array}$ \\
\hline $\begin{array}{l}1993 \text { Zabid \& Alsagoff: } \\
\text { JBE }\end{array}$ & 81: Malaysian managers & $\begin{array}{l}\text { Behavior of immediate supervisor most important } \\
\text { variable in influence to act unethically. }\end{array}$ \\
\hline 1994 Bruce: PPMR & 522: Managers & $\begin{array}{l}\text { Significant others more effective than code in } \\
\text { influencing ethical conduct. }\end{array}$ \\
\hline 1994 Grover \& Hui: JBE & 248: Students & $\begin{array}{l}\text { A relationship exists between significant others and } \\
\text { ethical decision-making in the organization. }\end{array}$ \\
\hline $\begin{array}{l}1994 \text { Soutar, McNeil \& } \\
\text { Molster: JBE }\end{array}$ & $\begin{array}{l}\text { 105: Western Australian } \\
\text { managers }\end{array}$ & $\begin{array}{l}\text { A relationship exists between significant others and } \\
\text { ethical decision-making in the organization. }\end{array}$ \\
\hline
\end{tabular}

while some evaluated singular issues with respect to awareness. Researchers fail to relate awareness to ethical behavior in organizations, representing a significant opportunity for study.

\section{F. Rewards and sanctions}

Rewards and sanctions, which are the major components of opportunity, were investigated in fifteen studies. A relationship between rewarding unethical behavior and the continuation of such behavior was revealed in a majority of these projects. Generally, rewarded and supported behaviors occur more frequently. Sanctions and their enforcement minimize opportunity. Additional research of rewards and sanctions should address communication issues at different levels in the organization, how other organizational members are informed of violations, and continuous improvement mechanisms for revising policies. 


\section{G. Significant others}

Eleven studies address significant others. Significant others (within the organization) greatly influence ethical decisions of their coworkers and peers as suggested by Ferrell and Gresham (1985). These studies show overwhelming support for the importance of managing relationships within the work group and the pervasive influence of peers in ethical decision making. Perhaps the greatest strides in understanding the influence of significant others can come from a better understanding of group decision making and the role of peer influence in ethical decision making. We know peers are influential, but is that influence greater in dayto-day decision making or with major ethics related decisions? When does the collectivity of the system break down and group members report behavior and decisions to other organizational members? Future research should address these questions.

\section{H. Miscellaneous categories}

The remaining areas of study provide very mixed findings (i.e., the role of nationality) or only a few studies addressing a particular issue (i.e., cognitive moral development in business, religion, locus of control, intent, general opportunity, and moral intensity). Areas such as moral intensity represent relatively new topics for investigation. This variable originated with Jones' (1991) synthesis model and additional research in this area is anticipated. Topics such as cognitive moral development and religion represent difficult areas of study to report and relate the findings to managerially actionable conclusions. Opportunity has often been assessed through the structural mechanisms within the organization (codes and policies, rewards and sanctions). Intent has proven to be a very difficult area to assess in organizational ethical decision making. And finally, locus of control relates to an individual's susceptibility to influence (external locus of control-particularly concerned with significant others versus an internal locus of control which indicates less susceptibility to peer influence).
Locus of control closely parallels, in ethical decision making, the concept of peer influencewhich has been extensively researched.

\section{Discussion and conclusions}

Organizational ethical decision making theory needs to be empirically tested to further our knowledge in the area of business ethics. Numerous researchers have contributed to our knowledge in this area through the testing of current ethical decision making models. A few researchers (i.e., Ford and Richardson, 1994; Tsalikis and Fritzsche, 1989) have provided helpful reviews of ethics research. This article reviews the empirical studies related to organizational ethical decision making theory and evaluates the general findings while observing areas that would benefit from further inquiry. Such an analysis is necessary for ethics researchers to evaluate the progress of knowledge development.

The tables provided offer a synopsis of the studies, their findings, samples researched, and the scope of the research. The Journal of Business Ethics published an overwhelming number of the studies (sixty-one), reaffirming the journal's status as the major outlet for business ethics research. Other outlets included the Journal of Business Research (eight) the Journal of Marketing Science (seven), and the Journal of Marketing (four). The paucity of articles published in other journals indicates a need to more thoroughly integrate ethics issues in other areas of research. Robin and Reidenbach (1987) indicate a need for the gap between ethics and marketing strategy to narrow. Further study integrating the ethical constructs suggested by ethical decision making theory with other marketing related variables, such as marketing orientation, quality and performance would be appropriate in narrowing the gap.

The studies reviewed here indicate a need to consider methodological issues when conducting ethics research. Longitudinal studies are necessary to more fully gain understanding of how ethical climate is impacted by ethics training and, as indicated above, how ethics constructs influence performance over time. Additional studies using industry samples is important to gaining 
face validity and in providing research results that will be given serious consideration by practitioners.

Understanding why and how individuals and groups make ethical decisions in a business context should improve the ethical decisions made in the organizational context. There is a difference between studying ethics in the personal lives of individuals and the ethical decisions made in organizations. People in organizations are influenced by the corporate culture and role relationships. While there is great difficulty in describing precisely how or why individuals in the work group make the decisions they do, ethical decision models attempt to generalize about the average or typical behavior patterns within organizations. The empirical studies undertaken should help managers who attempt to influence the ethical environment of their organizations through ethics training and compliance programs.

Though all of the constructs set forth in ethical decision making have been empirically examined, more research needs to be conducted on intent and moral intensity. This study chronicles research in business that has tested the major organizational ethical decision making constructs. These studies were related specifically to the constructs and relationships set forth in positive theories, which is one of the first steps to assess our overall level of understanding of organizational ethical decision making. Aristotle (1943) suggested that when such observations fail to support theory, the theory must be abandoned for theory that can be supported by empirical testing. Our efforts should assist in guiding the efforts of organizational ethics researchers.

\section{References}

Abratt, R., D. Nel and N. S. Higgs: 1992, 'An Examination of the Ethical Beliefs of Managers Using Selected Scenarios in a Cross-cultural Environment', Journal of Business Ethics 11, 29-35. Akaah, I.: 1989, 'Differences in Research Ethics Judgments Between Male and Female Marketing Professionals', Journal of Business Ethics 8, 375-381.
Akaah, I.: 1997, 'Influence of Deontological and Teleological Factors on Research Ethics Evaluations', Journal of Business Research 39, 71-80.

Alam, K. F.: 1993, 'Ethics in New Zealand Organisations', Journal of Business Ethics, 12, 433-440.

Allen, J. and D. Davis: 1993, 'Assessing Some Determinant Effects of Ethical Consulting Behavior. The Case of Personal and Professional Values', Journal of Business Ethics 12, 449-458.

Aristotle: 1943, Aristotle: On Man in the Universe, Edited by Louise Ropes Loomis (Gramercy Books, New York, New York).

Arlow, P. and T. A. Ulrich: 1980, 'Business Ethics, Social Responsibility and Business Students: An Empirical Comparison of Clark's Study', Akron Business and Economic Review 11(3), 17-23.

Armstrong, R. W.: 1996, 'The Relationship Between Culture and Perception of Ethical Problems in International Marketing', Journal of Business Ethics 15(11), 1199-1208.

Barnett, T.: 1992, 'A Preliminary Investigation of the Relationship Between Selected Organizational Characteristics and External Whistleblowing by Employees', Journal of Business Ethics 11, 949-959.

Barnett, J. H. and M. J. Karson: 1989, 'Managers, Values, and Executive Decisions: An Exploration of the Role of Gender, Career Stage, Organizational Level, Function, and the Importance of Ethics, Relationships and Results in Managerial Decision Making', Journal of Business Ethics 8, 747-771.

Barnett, T., D. Cochran and G. S. Taylor: 1993, 'The Internal Disclosure Policies of Private-Sector Employers: An Initial Look at Their Relationship to Employee Whistleblowing', Journal of Business Ethics 12, 127-136.

Baumhart, R.: 1961, 'How Ethical Are Businessmen?', Harvard Business Review 39(4), 6-19.

Becker, H. and D. J. Fritzche: 1987, 'Business Ethics: A Cross-cultural Comparison of Managers' Attitudes', Journal of Business Ethics 6, 289-295.

Bellizi, J. A. and R. E. Hite: 1989, 'Supervising Unethical Salesforce Behavior', Journal of Marketing 53, 36-47.

Beltramini, R. F., R. A. Peterson and G. Kozmetsky: 1984, 'Concerns of College Students Regarding Business Ethics', Journal of Business Ethics 3, 195-200.

Beneish, M. B. and R. Chatov: 1993, 'Corporate Codes of Conduct: Economic Determinants and Legal Implications for Independent Auditors', Journal of Accounting and Public Policy 12, 3-35.

Betz, M., L. O'Connell and J. M. Shepherd: 1989, 
'Gender Differences in Proclivity for Unethical Behavior', Journal of Business Ethics 8, 321-324.

Borkowski, S. and Y. Ugras: 1992, 'The Ethical Attitudes of Students as a Function of Age, Sex, and Experience', Journal of Business Ethics 11(12), 961-979.

Brady, F. N. and G. E. Wheeler 1996, 'An Empirical Study of Ethical Predispositions', Journal of Business Ethics 15, 927-940.

Brenner, S. N. and E. A. Molander: 1977, 'Is the Ethics of Business Changing?', Harvard Business Review 55(1), 57-71.

Browning, J. and N. B. Zabriskie: 1983, 'How Ethical are Industrial Buyers?’, Industrial Marketing Management 12, 219-224.

Bruce, W.: 1994, 'Ethical People Are Productive People', Public Productivity \& Management Review 17(3), 241-252.

Callahan, E. S. and J. W. Collins: 1992, 'Employee Attitudes Toward Whistleblowing: Management and Public Policy Implications', Journal of Business Ethics 11, 939-948.

Callan, V. J.: 1992. 'Predicting Ethical Values and Training Needs in Ethics', Journal of Business Ethics 11, 761-769.

Chonko, L. B. and S. D. Hunt: 1985, 'Ethics and Marketing Management: An Empirical Examination', Journal of Business Research 13 (August), 339-359.

Cohen, J., L. Pant, and D. Sharp: 1993, 'A Validation and Extension of a Multidimensional Ethics Scale', Journal of Business Ethics 12, 13-26.

Cyriac, K. and R. Dharmaraj: 1994, 'Machiavellianism in Indian Management', Journal of Business Ethics 13, 281-286.

Dabholkar, P. A. and J. J. Kellaris: 1992, 'Toward Understanding Marketing Students' Ethical Judgment of Controversial Personal Selling Practices', Journal of Business Research 25, 103109.

Dawson, L. M.: 1992, 'Will Feminization Change the Ethics of the Sales Profession?', Journal of Personal Selling and Sales Management 12(1), 21-32.

DeConinck, J. B.: 1992, 'How Sales Managers Control Unethical Sales Force Behavior', Journal of Business Ethics 11, 789-798.

Derry, R.: 1989, 'An Empirical Study of Moral Reasoning Among Managers', Journal of Business Ethics 8, 855-862.

Dubinsky, A. J. and J. M. Gwin: 1981, 'Business Ethics: Buyers and Sellers', Journal of Purchasing and Materials Management (Winter), 97-103.

Dubinsky, A. J. and T. N. Ingram: 1984, 'Correlates of Salespeople's Ethical Conflict: An Exploratory Investigation', Journal of Business Ethics 3, 343353.

Dubinsky, A. J., M. A. Jolson, A. Marvin, R. E. Michaels, M. Kotabe and C. U. Lim: 1992, 'Ethical Perceptions of Field Sales Personnel: An Empirical Assessment', Journal of Personal Selling and Sales Management 12(4), 9-21.

Dubinsky, A. J. and M. Levy: 1985, 'Ethics in Retailing Perceptions of Retail Salespeople', Journal of the Academy of Marketing Science 13(1), 1-16.

Dubinsky, A. J. and B. Loken: 1989, 'Analyzing Ethical Decision Making in Marketing', Journal of Business Research 19, 83-107.

Elm, D. R. and M. L. Nichols: 1993, 'An Investigation of the Moral Reasoning of Managers', Journal of Business Ethics 12, 817-833.

Ferrell, O. C and J. Fraedrich: 1991, 'Moral Philosophies of Marketing Managers: An Empirical Investigation', Journal of Marketing Management (Fall), 87-106.

Ferrell, O. C. and L. G. Gresham: 1985, 'A Contingency Framework for Understanding Ethical Decision Making in Marketing', Journal of Marketing 49 (Summer), 87-96.

Ferrell, O. C., L. G. Gresham and J. Fraedrich: 1989, 'A Synthesis of Ethical Decision Models for Marketing', Journal of Macromarketing (Fall), 55-64.

Ferrell, O. C. and S. J. Skinner: 1988, 'Ethical Behavior and Bureaucratic Structure in Marketing Research Organizations', Journal of Marketing Research 25, 103-109.

Ferrell, O. C. and M. K. Weaver: 1978, 'Ethical Beliefs of Marketing Managers', Journal of Marketing (July), 69-73.

Ford, R. C. and W. D. Richardson: 1994, 'Ethical Decision Making: A Review of the Empirical Literature', Journal of Business Ethics 13, 205-221.

Fraedrich, J. P.: 1993, 'The Ethical Behavior of Retail Managers', Journal of Business Ethics 12, 207-218.

Fraedrich, J. P. and O. C. Ferrell: 1992, 'Cognitive Consistency of Marketing Managers in Ethical Situations', Journal of the Academy of Marketing Science 20(3), 245-252.

Fraedrich, J. P. and O. C. Ferrell: 1992, 'The Impact of Perceived Risk and Moral Philosophy Type on Ethical Decision Making in Organizations', Journal of Business Research 24, 283-295.

Galbraith, S. and H. B. Stephenson: 1993, 'Decision Rules Used by Male and Female Business Students in Making Ethical Value Judgements: Another Look', Journal of Business Ethics 12, 227-233.

Glenn, J.R., Jr. and M. F. Van Loo: 1993, 'Business 
Students' and Practitioners' Ethical Decisions Over Time', Journal of Business Ethics 12, 835-847.

Goodman, C. S. and C. M. Crawford: 1974, 'Young Executives: A Source of New Ethics?', Personnel Journal (March), 180-187.

Goolsby, J. R. and S. D. Hunt: 1992, 'Cognitive Moral Development and Marketing', Journal of Marketing 56(1), 55-68.

Grover, S. L. and C. Hui: 1994, 'The Influence of Role Conflict and Self-Interest on Lying in Organizations', Journal of Business Ethics 13, 293-303.

Hawkins, D. I. and A. B. Cocanougher: 1972, 'Student Evaluations of the Ethics of Marketing Practices: The Role of Marketing Education', Journal of Marketing 36 (April), 61-64.

Hegarty, W. H. and H. P. Sims, Jr.: 1978, 'Some Determinants of Unethical Decision Behavior: An Experiment', Journal of Applied Psychology 63, 451-457.

Henthorne, T. L., D. P. Robin and R. E. Reidenbach: 1992, 'Identifying the Gaps in Ethical Perceptions Between Managers and Salespersons: A Multidimensional Approach', Journal of Business Ethics 11, 849-856.

Hunt, S. D.: 1991, Modern Marketing Theory: Critical Issues in the Philosophy of Marketing Science (SouthWestern Publishing Company, Cincinnati, $\mathrm{OH}$ ).

Hunt, S. D., P. L. Kiecker and L. B. Chonko: 1990, 'Social Responsibility and Personal Success: A Research Note', Journal of the Academy of Marketing Science 18(3), 239-244.

Hunt, S. D and A. Z. Vasquez-Parraga: 1993, 'Organizational Consequences, Marketing Ethics, and Salesforce Supervision', Journal of Marketing Research 30 (February), 78-90.

Hunt, S. D and S. Vitell: 1986, 'A General Theory of Marketing Ethics', Journal of Macromarketing 6 (Spring), 5-16.

Izraeli, D.: 1988, 'Ethical Beliefs and Behavior Among Managers: A Cross-cultural Perspective', Journal of Business Ethics 7, 263-271.

Jones, T. M.: 1991, 'Ethical Decision Making by Individuals in Organizations: An Issue-Contingent Model', Academy of Management Review 16(2), 366-395.

Jones, T. M. and F. J Gautschi, III: 1988, 'Will the Ethics of Business Change? A Survey of Future Executives', Journal of Business Ethics 7, 231-248.

Jones, S. K. and K. M. Hiltebeitel: 1995, 'Organizational Influence in a Model of the Moral Decision Process of Accountants', Journal of Business Ethics 14, 417-431.
Judge, W. Q., Jr.: 1994, 'Correlates of Organizational Effectiveness: A Multilevel Analysis of a Multidimensional Outcome', Journal of Business Ethics 13, $1-10$.

Kawathatzopoulos, I.: 1993, 'Development of a Cognitive Skill in Solving Business Ethics Problems: The Effect of Instruction', Journal of Business Ethics 12, 379-386.

Kaye, B. N.: 1992, 'Codes of Ethics in Australian Business Corporations', Journal of Business Ethics 11, 857-862.

Kelley, S. W., O. C. Ferrell and S. J. Skinner: 1990, 'Ethical Behavior Among Marketing Researchers: An Assessment of Selected Demographic Characteristics', Journal of Business Ethics 9, 681-688.

Kidwell, J. M., R. E. Stevens and A. L. Bethke: 1987, 'Differences in the Ethical Perceptions Between Male and Female Managers: Myth or Reality', Journal of Business Ethics 6, 489-493.

Klebe-Trevino, L. and S. A. Youngblood: 1990, 'Bad Apples in Bad Barrels: A Causal Analysis of Ethical Decision making Behavior', Journal of Applied Psychology 75(4), 378-385.

'Kohut, G. F. and S. E. Corriher: 1994, 'The Relationship of Age, Gender, Experience and Awareness of Written Ethics Policies to Business Decision Making', SAM Advanced Management Journal, 32-39.

Laczniak, G. and E. J. Inderrieden: 1987, 'The Influence of Stated Organizational Concern upon Ethical Decision Making', Journal of Business Ethics 6, 297-307.

Lane, M. S., D. Schaupp and B. Parsons: 1988, 'Pygmalion Effect: An Issue for Business Education and Ethics', Journal of Business Ethics 7, 223229.

LaFleur, E. K., R. E. Reidenbach, D. P. Robin and P. J. Forrest: 1996, 'An Exploration of Rule Configuration Effects on the Ethical Decision Processes of Advertising Professionals', Journal of the Academy of Marketing Science 24(1), 66-76.

McCabe, D. L., L. K. Trevino and K. D. Butterfield: 1996, 'The Influence of Collegiate and Corporate Codes of Conduct on Ethics-Related Behavior in the Workplace', Business Ethics Quarterly 6(4), 461-476.

McDonald, G. and P. C. Pak: 1996, 'It's All Fair in Love, War, and Business: Cognitive Philosophies in Ethical Decision Making', Journal of Business Ethics 15, 973-996.

Mayo, M. A. and L. J. Marks: 1990, 'An Empirical Investigation of a General Theory of Marketing 
Ethics', Journal of the Academy of Marketing Science 18, 163-171.

McNichols, C. W. and T. W. Zimmerer: 1985, 'Situational Ethics: An Empirical Study of Differentiators of Student Attitudes', Journal of Business Ethics 4, 175-180.

Morgan, R. B.: 1993, 'Self and Co-Worker Perceptions of Ethics and Their Relationships to Leadership and Salary', Academy of Management Journal 36(1), 200-214.

Muncy, J. and S. Vitell: 1992, 'Consumer Ethics: An Investigation of the Ethical Beliefs of the Final Consumer', Journal of Business Research 24, 297-311.

O'Clock, P. and M. Okleshen: 1993, 'A Comparison of Ethical Perceptions of Business', Journal of Business Ethics 12, 677-687.

Posner, B. and W. Schmidt: 1993, 'Value Congruence and Differences Between the Interplay of Personal and Organizational Value Systems', Journal of Business Ethics 12, 341-347.

Reidenbach, R. E. and D. P. Robin: 1990, 'A Partial Testing of the Contingency Framework for Ethical Decision Making: A Path Analytical Approach', Southern Marketing Association Proceedings, 121128.

Rest, J. R.: 1986, Moral Development: Advances in Research and Theory (Praeger, New York, NY).

Robertson, D. C. and B. B. Schlegelmilch: 1993, 'Corporate Institutionalization of Ethics in the United States and Great Britain', Journal of Business Ethics 12, 301-312.

Robin, D. P. and R. E. Reidenbach: 1987, 'Social Responsibility, Ethics, and Marketing Strategy: Closing the Gap Between Concept and Application', Journal of Marketing 51 (January), 44-58.

Robin, D. P., R. E. Reidenbach and P J. Forrest: 1996, 'The Perceived Imporance of an Ethical Issue as an Influence on the Ethical Decision making of Ad Managers', Journal of Business Research 35, 17-28.

Ruegger, D. and E. W. King: 1992, 'A Study of the Effect of Age and Gender Upon Student Business Ethics', Journal of Business Ethics 11, 179-186.

Schultz, J. J., Jr., D. A. Johnson, D. Morris and A. Dyrnes: 1993, 'An Investigation of the Reporting of Questionable Acts in an International Setting', Journal of Accounting Research 31 (Supplement), 75-103.

Schwepker, C. K. Jr., O. C. Ferrell and T. N. Ingram: 1997, 'The Influence of Ethical Climate and Ethical Conflict on Role Stress in the Sales Force',
Journal of the Academy of Marketing Science 25(2), 99-108.

Serwinek, P. J.: 1992, 'Demographic and Related Differences in Ethical Views Among Small Businesses', Journal of Business Ethics 11, 555-566.

Shaub, M. K., D. W. Finn and P. Munter: 1993, 'The Effects of Auditors' Ethical Orientation on Commitment and Ethical Sensitivity', Behavioral Research in Accounting 5, 145-169.

Simpson, P. M., D. Banerjee and C. L. Simpson, Jr.: 1994, 'Softlifting: A Model of Motivating Factors', Journal of Business Ethics 13, 431-438.

Singhapakdi, A. and S. J. Vitell: 1990, 'Marketing Ethics: Factors Influencing Perceptions of Ethical Problems and Alternatives', Journal of Macromarketing, 47-65.

Singhapakdi, A. and S. J. Vitell: 1993, 'Personal and Professional Values Underlying the Ethical Judgements of Marketers', Journal of Business Ethics 12, 525-533.

Singhapakdi, A., S. J. Vitell and K. L. Kraft: 1996, 'Moral Intensity and Ethical Decision Making of Marketing Professionals', Journal of Business Research 36, 245-255.

Small, M. W.: 1992, 'Attitudes Towards Business Ethics Held by Western Australian Students: A Comparative Study', Journal of Business Ethics 11, 745-752.

Soutar, G., M. M. McNeil and C. Molster: 1994, 'The Impact of the Work Environment on Ethical Decision Making: Some Australian Evidence', Journal of Business Ethics 13, 327-339.

Sridhar, B. S. and A. Camburn: 1993, 'Stages of Moral Development of Corporations', Journal of Business Ethics 12, 727-739.

Stevens, G. E.: 1984, 'Business Ethics and Social Responsibility: The Response of Present and Future Managers', Akron Business and Economic Review (Fall), 6-11.

Stevens, G. E., W. D. Richardson and A. E. Abramowitz: 1989, 'Perceptual Differences of Ethical Decision Situations Business vs. Law: A Difference of Opinion?', Southern Management Association Proceedings, 199-201.

Stevens, R., J. Harris and S. Williamson: 1993, 'A Comparison of Ethical Evaluations of Business School Faculty and Students: A Pilot Study', Journal of Business Ethics 12(8), 611-619.

Swinyard, W. R., T. J. DeLong and P. S. Cheng: 1989, 'The Relationship Between Moral Decisions and Their Consequences: A Tradeoff Analysis Approach', Journal of Business Ethics 8, 289-297.

Tansey, R. G., H. Brown, M. R. Hyman and L. E. 
Dawson, Jr.: 1994, 'Personal Moral Philosophies and the Moral Judgements of Salespeople', Journal of Personal Selling and Sales Management 14(1), 59-75.

Thorne, D. M. and O. C. Ferrell: 1993, 'Assessing the Application of Cognitive Moral Development Theory to Business Ethics', Journal of Business Ethics 10, 829-838.

Trevino, L. K.: 1986, 'Ethical Decision Making in Organizations: A Person-Situation Interactionist Model', Academy of Management Review, 601-617.

Trevino, L. K and B. Victor: 1992, 'Peer Reporting of Unethical Behavior: A Social Context Perspective', Academy of Management Journal 35(1), 38-64.

Trevino, L. K and S. A. Youngblood: 1990, 'Bad Apples in Bad Barrels: A Causal Analysis of Ethical Decision Making Behavior', Journal of Applied Psychology 75(4), 378-385.

Tsalikis, J. and D. J. Fritsche: 1989, 'Business Ethics: A Literature Review with a Focus on Marketing Ethics', Journal of Business Ethics 18, 695-753.

Tyson, T.: 1992, 'Does Believing that Everyone Else is Less Ethical have an Impact on Work Behavior?', Journal of Business Ethics 11, 707-717.

Verbeke, W., C. Ouwerkerk and E. Peelen: 1996, 'Exploring the Contextual and Individual Factors on Ethical Decision Making of Salespeople', Journal of Business Ethics 15, 1175-1187.

Victor, B. and J. B. Cullen: 1988, 'The Organizational Bases of Ethical Work Climate', Administrative Science Quarterly 33(1), 101-125.

Victor, B., L. K. Trevino and D. L. Shapiro: 1993, 'The Influence of Justice Evaluations and Social Context Factors', Journal of Business Ethics 12(4), 253-263.

Vitell, S, K. C. Rallapalli and A. Singhapakdi: 1993, 'Marketing Norms: The Influence of Personal Moral Philosophies and Organizational Ethical Climate', Journal of the Academy of Marketing Science 21(4), 331-337.

Wahn, J.: 1993, 'Organizational Dependence and the Likelihood of Complying with Organizational Pressures to Behave Unethically', Journal of Business Ethics 12, 245-251.

Wang, J. and B. S. Coffey: 1992, 'Board Composition and Corporate Philanthropy', Journal of Business Ethics 11, 771-778.

Weaver, K. M. and O. C. Ferrell: 1977, 'The Impact of Corporate Policy in Reported Ethical Beliefs and Behavior of Marketing Practitioners', $A M A$ Proceedings, 477-481.
Weber, J.: 1990, 'Managers' Moral Reasoning: Assessing Their Responses to Three Moral Dilemmas', Human Relations 43(7), 687-702.

Whipple, T. W. and D. F. Swords: 1992, 'Business Ethics Judgments: A Cross-cultural Comparison’, Journal of Business Ethics 11, 671-678.

White, C. S. and R. S. Dooley: 1993, 'Ethical or Practical: An Empirical Study of Students' Choices in Simulated Business Scenarios', Journal of Business Ethics 12, 643-651.

White, L. P. and M. J. Rhodeback: 1992, 'Ethical Dilemmas in Organization Development: A CrossCultural Analysis', Journal of Business Ethics 11, 663-670.

Wimalasiri, J. S., F. Pavri and A. A. K. Jalil: 1996, 'An Empirical Study of Moral Reasoning Among Managers in Singapore', Journal of Business Ethics 15, 1331-1341.

Zabid, A. R. M. and S. K. Alsagoff: 1993, 'Perceived Ethical Values of Malaysian Managers', Journal of Business Ethics 12, 331-337.

Zahra, S. A.: 1989, 'Executive Values and the Ethics of Company Politics: Some Preliminary Findings', Journal of Business Ethics 8, 15-29.

Zey-Ferrell, M. and O. C. Ferrell: 1982, 'Role-Set Configuration and Opportunity as Predictors of Unethical Behavior in Organizations', Human Relations 35(7), 587-604.

Zey-Ferrell, M., K. M. Weaver and O. C. Ferrell: 1979, 'Predicting Unethical Behavior Among Marketing Practitioners', Human Relations 32(7), $557-569$

Terry W. Loe Department of Marketing, Hankamer School of Business, Baylor University, P.O. Box 98007, Waco, TX 76798-8007, U.S.A.

Linda Ferrell University of Tampa, 401 W. Kennedy, Tampa, FL 33606.

Phylis Mansfield Andrews University, Department of Management and Marketing, Berrien Springs, MI 49104. 